\title{
AUSENCIA DE TRANSMISIÓN PERINATAL DE VIH EN 40 EMBARAZADAS TRATADAS CON TERAPIA ANTT-REIROVIRAL DE ALTA POTENCIA*
}

\author{
Fernando Abarzúa C. ${ }^{1,3}$, Carlos Pérez C. ${ }^{2}$, Claudio Callejas C. ${ }^{2}$, Jean Cyr Jombi ${ }^{3}$, \\ Bernard Vandercam ${ }^{3}$
}

${ }^{1}$ Departamento de Obstetricia y Ginecología, ${ }^{2}$ Departamento de Medicina Interna, Pontificia Universidad Católica de Chile. ${ }^{3}$ Departamento de Medicina Interna e Infectología y Enfermedades Tropicales, Cliniques Universitaires Saint Luc, Université Catolique de Lovaine

aInterno de Medicina, Universidad Católica de Chile

\section{RESUMEN}

Objetivo. Evaluar la magnitud de la transmisión perinatal de VIH, en embarazadas infectadas tratadas con terapia anti-retroviral de alta potencia y los efectos secundarios materno-perinatal. Pacientes y método. Se estudian 40 embarazadas VIH (+) controladas en las Universidades Católicas de Chile y de Lovaina, Bélgica, en el período 1999 - 2003. Todas recibieron terapia anti-retroviral de alta potencia. Se les permitió parto vaginal a las que tenían carga viral menor a 1000 copias $/ \mathrm{ml}$ al final del tercer trimestre. Se determinó la presencia de infección en el recién nacido, mediante técnica de PCR, con un seguimiento mínimo de 6 meses. Resultados. El 70\% inició tratamiento a las 24 semanas de gestación. No hubo efectos adversos que requiriesen suspensión del tratamiento. El 30\% tenía carga viral menor a 1000 copias $/ \mathrm{ml}$ en el primer trimestre aumentando a $97,5 \%$ al momento del parto con la terapia. No hubo casos de transmisión perinatal (seguimiento 6 meses - 3 años). Hubo una muerte neonatal por prematurez (27 semanas). El 50\% de las pacientes tuvo parto vaginal. Conclusión. La terapia antiretroviral de alta potencia logró una efectiva profilaxis de la transmisión perinatal (cero por ciento de TPN en nuestra serie). El parto vaginal en aquellas que tienen carga viral menor de 1000 copias $/ \mathrm{ml}$ no modificó ese riesgo. No hubo efectos adversos significativos por el tratamiento.

\section{PALABRAS CLAVE: Embarazo, VIH, terapia anti-retroviral}

\section{SUMMARY}

Objective. Assess the rate of perinatal transmission of HIV, in pregnant patients who receive highly active antiretroviral therapy (HAART), and maternal and perinatal side effects. Patients and method. Forty pregnant women with HIV infection attended during 1999 to 2003 period at Catholic University of Chile and Saint Luc University Hospital of Belgium are included. All of them received HAART therapy. A vaginal delivery was proposed only for patients who had a viral load of 1000 copies $/ \mathrm{ml}$ or less at end of third trimester. The presence of HIV infection in newborns and infants was detected by PCR. Newborns had a follow up of 6 month as minimum. Results. $70 \%$ of patients started the therapy at 24 weeks of gestation. There were not serious side effects, enough to suspend the therapy. Only a $30 \%$ had a viral load less than 1000 copies $/ \mathrm{ml}$ at the first trimester and increased to $95 \%$ at delivery with HAART. There

\footnotetext{
* Trabajo leído en la sesión del martes 7 de septiembre de 2004 de la Sociedad Chilena de Obstetricia y Ginecología.
} 
were not cases of perinatal transmission of HIV (follow up from 6 months to 3 years). There was a case of neonatal death by prematurity (27 weeks). Fifty percent of patients had a vaginal delivery. Conclusions. HAART therapy is a successful prophylaxis to perinatal transmission (cero percent in our experience). The risk is not modified with vaginal delivery in patients with viral load of 1000 copies $/ \mathrm{ml}$ or less at delivery. Not important side effects were observed.

\section{KEY WORDS: Pregnancy, VIH, antiretroviral therapy, HAART}

\section{INTRODUCCIÓN}

Ya han pasado más de dos décadas desde la notificación del primer caso de síndrome de inmunodeficiencia adquirida (1). Desde ese momento se han producido cambios epidemiológicos notables en esta epidemia. Uno de ellos es el paso de una enfermedad predominantemente del sexo masculino a un aumento proporcional de casos en mujeres y de infección de adquisición perinatal. En relación con el diferente acceso a los avances logrados en el tratamiento, los pacientes pertenecientes a países desarrollados acceden a terapia anti-retroviral de alta potencia, que si bien es de alto costo y no exenta de efectos adversos, ha transformado la infección en una enfermedad crónica, disminuyendo las infecciones oportunistas y las hospitalizaciones, reduciendo significativamente el riesgo de transmisión perinatal, esto permite una visión diferente de la maternidad en mujeres infectadas $(2,3)$, mientras que en los países en vías de desarrollo y menos desarrollados esos avances no se han podido incorporar.

Chile está en una situación intermedia. Las embarazadas infectadas atendidas en el sistema público tienen acceso a terapia bi-asociada, y lentamente se registra un aumento del número de mujeres que acceden a terapia anti-retroviral de alta potencia, principalmente en el sector privado.

El objetivo de este estudio es analizar la experiencia conjunta de embarazadas infectadas expuestas a terapia anti-retroviral de alta potencia controladas y tratadas en dos hospitales universitarios: el Hospital Clínico de la Universidad Católica de Chile y la Universidad Católica de Lovaina (Cliniques Saint Luc, Bruselas, Bélgica).

\section{MATERIAL Y MÉTODO}

Se estudian embarazadas infectadas por virus de la inmunodeficiencia humana (VIH), que controlaron sus embarazos en el período 1999 - 2003, en el Hospital Clínico de la Universidad Católica de Chile ( 5 pacientes) y en el Hospital de la Universidad Católica de Lovaina (35 pacientes). To- das fueron tratadas con terapia anti-retroviral de alta potencia durante la gestación. Se define terapia anti-retroviral de alta potencia al uso de tres drogas anti-retrovirales (tri-terapia).

Se describen las características inmunológicas según clasificación del Centro de Control de Enfermedades de Estados Unidos (4), y la evolución de ésta durante el embarazo. Se describe el tipo de terapia anti-retroviral de alta potencia recibida y la edad gestacional de exposición al tratamiento. Se describe la evolución de la carga viral determinada por técnica ultrasensible (umbral 30 copias/ $\mathrm{ml}$ ). Se analizan los efectos secundarios de la terapia, las complicaciones obstétricas, las características del parto y el resultado perinatal, incluida la transmisión perinatal (TPN) de VIH.

La vía de resolución del embarazo fue decidida considerando la carga viral cercana al parto, de acuerdo a la recomendación del Colegio Americano de Obstetricia y Ginecología (ACOG) (5), y los antecedentes obstétricos. El parto vaginal fue permitido a las con carga viral inferior a 1000 copias/ $\mathrm{ml}$ o indetectable, y sin contraindicación obstétrica a esa vía, conservando las membranas íntegras el mayor tiempo posible; se efectuó cesárea cuando el intervalo estimado entre la rotura de las membranas y el parto fuera superior a $4-6$ horas.

\section{RESULTADOS}

En el período 1999-2003, 40 mujeres infectadas por $\mathrm{VIH}$, fueron tratadas con terapia antiretroviral de alta potencia durante el embarazo. Las características generales se presentan en la Tabla I.

Según la clasificación del CDC, 29 pacientes $(72,5 \%)$ pertenecían al grupo A (asintomáticas), 8 $(20 \%)$ al grupo B (otras enfermedades asociadas a $\mathrm{VIH}$, no constitutivas de SIDA), y $3(7,5 \%)$ al grupo C (enfermedades oportunistas, infecciosas o neoplásicas que hacen el diagnóstico de SIDA). En catorce pacientes (35\%) el diagnóstico de infección VIH se realizó al solicitar el examen en el primer control prenatal del embarazo actual o previo, $26(65 \%)$ ya conocían su estado de infectadas 
Tabla I

\section{CARACTERÍSTICAS GENERALES DE LAS PACIENTES EN ESTUDIO}

\begin{tabular}{lc}
\hline Características & $n$ \\
\hline Pacientes & 40 \\
Edad promedio (años) y rango & $31,1(22-43)$ \\
Alcoholismo & $1(2,5 \%)$ \\
Hipertensión crónica & $2(5 \%)$ \\
Herpes genital recurrente & $2(5 \%)$ \\
Epilepsia & $1(2,5 \%)$ \\
Otras & $7(17 \%)$ \\
\hline
\end{tabular}

al momento de embarazarse. El recuento de linfocitos CD4 en el primer trimestre fue en promedio de 404 (R: 50 - 720), observándose un aumento en el tercer trimestre a 458 (R: 168 - 917). El $30 \%$ de las pacientes tenían carga viral no detectable o muy baja (<1.000 copias/ml) en el primer trimestre, subiendo a $97,5 \%$ en el tercero, diferencia estadísticamente significativa (Tabla II). Todas las pacientes llegaron al parto con carga viral indetectable o inferior a $1.000 \mathrm{copias} / \mathrm{ml}$, con excepción de una paciente que fue tratada con triterapia previo al embarazo, y que suspendió el tratamiento por fracaso virológico antes de embarazarse; desde las 26 semanas reinició un nuevo esquema de acuerdo al patrón de resistencia, con una carga viral de 187.000 copias/ml y que llegó con 1.920 copias/ml al momento del parto (38 semanas) (descenso adecuado desde punto de

Tabla II

\section{ASPECTOS VIROLÓGICOS E INMUNOLÓGICOS DE LAS PACIENTES}

\begin{tabular}{lrc}
\hline Estadío* $^{*}$ & $n$ & $\%$ \\
\hline A & 29 & 72,5 \\
B & 8 & 20,0 \\
C & 3 & 7,5 \\
Diagnóstico de infección por VIH & & \\
Durante este embarazo & 10 & 25 \\
Durante otro embarazo & 4 & 10 \\
Sin embarazo & 26 & 65 \\
Pacientes con carga viral $\leq 1000$ & & \\
Primer control prenatal & 12 & 30,0 \\
Ultimo control antes del parto & 39 & 97,5 \\
Linfocitos CD4 & & \\
Primer control prenatal & 404,1 & Rango \\
Ultimo control antes del parto & 458,1 & $168-917$ \\
\hline
\end{tabular}

"Según clasificación del CDC, 2002. vista virológico). El primer control de carga viral en el postparto fue indetectable.

Dieciocho pacientes (45\%) nunca habían recibido terapia anti-retroviral (naive). Las 22 pacientes restantes (55\%) tenían ya experiencia con diversos esquemas; de éstas, 7 (31,8\%) mantuvieron su terapia durante todo el período de gestación, y $15(68,1 \%)$ lo suspendieron previo a intentar embarazo o inmediatamente conocido su estado de embarazadas. Todas recibieron terapia antiretroviral de alta potencia en la segunda mitad de la gestación y 7 fueron expuestas durante los tres trimestres. De las 33 pacientes restantes, ya sea porque suspendieron o nunca habían recibido terapia, 28 (70\% del total de pacientes) iniciaron la terapia alrededor de las 24 - 25 semanas (Tabla III).

Todas las pacientes recibieron terapia antiretroviral combinada de alta potencia, con esquemas similares a los utilizados en pacientes no embarazadas (con excepción de efavirenz, por su

Tabla III

CARACTERÍSTICAS DE LA TERAPIA ANTIRETROVIRAL DE ALTA POTENCIA (HAART)

Experiencia con terapia antiretroviral previa al embarazo

No ("naive")

$\mathrm{Si}$

- Mantiene terapia (\% de $n=22$ )

- Suspende transitoriamente la

terapia (\% de $\mathrm{n}=22$ )

HAART durante embarazo según trimestre de exposición

|-II-III

II-III

III ( $\geq 24$ semanas)

Esquemas utilizados INTR-INNTR INTR-IP

Drogas más utilizadas INTR

- Zidovudina

- Lamivudine (3TC)

$28 \quad 70$

INNTR

- Nevirapine $36 \quad 90$

IP

- Indinavir

1230

- Nelfinavir

$13 \quad 33$

INTR: Inhibidor nucleosídico transcriptasa reversa; INNTR: Inhibidor no nucleosídico transcriptasa reversa; IP: Inhibidor de proteasa. 
conocido efecto teratogénico en animales), privilegiando el uso de zidovudine (AZT) dentro del esquema. La principal combinación fue el uso de dos inhibidores de transcriptasa reversa del tipo nucleósido (INTR) más un inhibidor de proteasa (28 pacientes, 70\%), y luego dos INTR más un inhibidor no nucleosídico de transcriptasa reversa (INNTR), habitualmente zidovudine + lamivudine + nevirapine; veintisiete pacientes $(67,5 \%)$ recibieron zidovudine dentro de su esquema (Tabla III).

En la Tabla IV se presentan los efectos secundarios del tratamiento que obligó a efectuar cambios de la terapia. Tres pacientes por intolerancia digestiva (diarrea y/o vómitos), dos del grupo INTR+IP y una de INTR+INNTR; una por exantema extenso atribuido a indinavir, que cedió al cambiar a nelfinavir; otra por cuadro colestásico con prurito severo que disminuyó significativamente al suspender zidovudine que fue reemplazada por stavudine; otra presentó hepatitis con marcadores virales negativos, que motivó la suspensión de nevirapine y su cambio por nelfinavir. No hubo casos de acidosis láctica ni otros cuadros sugerentes de toxicidad mitocondrial.

En la Tabla V se presentan las complicaciones obstétricas. Dos pacientes (5\%) presentaron parto prematuro, una por el desarrollo de síndrome de HELLP, que fue interrumpida a las 27 semanas de gestación; el recién nacido pesó 720 g, falleciendo al segundo día de vida por hemorragia intracraneana, con carga viral negativa en sangre de cordón y al segundo día de vida. El segundo caso correspondió a una paciente tratada con 2INTR+1INNTR durante todo el embarazo y carga viral indetectable, que ingresó en trabajo de parto espontáneo a las 36 semanas, en presentación de nalgas, asistido sin incidentes por vía vaginal; el recién nacido pesó $2600 \mathrm{~g}$, con seguimiento al primer año de vida sin evidencias de infección. Dos pacientes $(5 \%)$ desarrollaron preeclampsia, la primera, ya descrita (HELLP), y la segunda correspondió a

Tabla IV

EFECTOS SECUNDARIOS DE LA TERAPIA QUE REQUIRIÓ MODIFICAR ESQUEMA DE TRATAMIENTO

\begin{tabular}{lcc}
\hline Efecto & $n$ & $\%$ \\
\hline Intolerancia digestiva & 3 & 7,5 \\
Rash & 1 & 2,5 \\
Prurito & 1 & 2,5 \\
Hepatitis & 1 & 2,5 \\
\hline Total & 6 & 15,0
\end{tabular}

Tabla V

\section{COMPLICACIONES OBSTÉTRICAS EN PACIENTES EXPUESTAS A TRI-TERAPIA EN EL EMBARAZO}

\begin{tabular}{llc}
\hline Complicación & $n$ & $\%$ \\
\hline Parto prematuro & 2 & 5 \\
Pre-eclampsia & 2 & 5 \\
Diabetes gestacional & 3 & 7,5 \\
Colestasia intrahepática & 1 & 2,5 \\
Oligohidramnios & 1 & 2,5 \\
\hline Total & 9 & 22,5 \\
\hline
\end{tabular}

una paciente hipertensa crónica con preeclampsia sobreagregada (37 1/2 semanas), tenía carga viral indetectable al momento del parto, se interrumpió por cesárea por malas condiciones obstétricas; el recién nacido pesó $3.750 \mathrm{~g}$, sin evidencias de infección al año de vida. Tres pacientes presentaron diabetes gestacional; todas tenían esquemas con inhibidores de proteasa, con buen control metabólico con dieta; una paciente presentó un cuadro de colestasia intrahepática en el tercer trimestre, que mejoró significativamente con el cambio de la terapia anti-retroviral antes mencionada.

Veinte pacientes $(50 \%)$ resolvieron su parto por vía vaginal, en dos se realizó episiotomía. Sólo una de veinte tuvo un intervalo entre la rotura de membranas y el parto superior a 4 horas. En las veinte pacientes restantes se realizó operación cesárea, todas con un intervalo entre la rotura de las membranas y el parto inferior a 4 horas (Tabla $\mathrm{VI})$.

No hubo casos de transmisión perinatal de infección por VIH en la serie. Todos los niños tuvieron un seguimiento de al menos 6 meses; el más prolongado fue de 5 años. La tasa de prematurez fue de $5 \%$ (2 casos), hubo un caso de mortalidad perinatal $(2,5 \%)$. Tres niños fueron pequeños $(7,5 \%)$ y seis $(15 \%)$ grandes para la edad gestacional, ninguno era hijo de madre diabética (Tabla VII)

Tabla VI

CARACTERÍSTICAS DEL PARTO EN PACIENTES EXPUESTAS A TRI-TERAPIA EN EL EMBARAZO

\begin{tabular}{lrc}
\hline Característica & $n$ & $\%$ \\
\hline Vaginal & 20 & 50 \\
Cesárea & 20 & 50 \\
Rotura de membranas $>4$ horas & 1 & 2,5 \\
\hline
\end{tabular}


Tabla VII

\section{RESULTADO PERINATAL EN NIÑOS EXPUESTOS IN ÚTERO A TRI-TERAPIA}

\begin{tabular}{llr}
\hline Resultado perinatal & $n$ & $\%$ \\
\hline Transmisión perinatal & 0 & 0,0 \\
Prematurez & 2 & 5,0 \\
Restricción del crecimiento fetal & 3 & 7,5 \\
Macrosomía & 6 & 15,0 \\
Mortalidad perinatal & 1 & 2,5 \\
\hline
\end{tabular}

\section{DISCUSIÓN}

En 1994 se demostró que la administración de zidovudina podía reducir la transmisión maternofetal del virus HIV- 1 en casi el $70 \%$ comparado a un grupo placebo (6). Posteriormente, el manejo de las embarazadas infectadas ha mejorado teniendo en cuenta los siguientes elementos:

- Un mejor conocimiento de la patogénesis de la transmisión perinatal, que tiene lugar esencialmente al final del tercer trimestre 0 durante el parto (7).

- La incorporación de la tri-terapia, que es considerada actualmente como el tratamiento estándar (2), que permite una mejor reconstitución inmunitaria y un mejor control de la replicación viral con relación a la monoterapia con AZT e incluso comparado con la biterapia, lo que se asocia a reducción del riesgo de seleccionar un virus resistente a los anti-retrovirales, evitando comprometer opciones terapéuticas posteriores (8).

- Evidencia que indica que la tri-terapia es el esquema con mayor reducción de la transmisión perinatal de VIH (8 -10).

Este estudio describe los resultados de cuarenta pacientes embarazadas, que si bien se controlaron en dos centros de países distintos, compartían varios elementos en común. El primero y motivo del trabajo es que fueron tratadas con triterapia. Los criterios de manejo de la infección VIH y del embarazo son similares en ambos centros, y las pacientes fueron tratadas por equipos multidisciplinarios.

En esta serie la mayoría de las pacientes $(72,5 \%)$ estaban en categoría $\mathrm{A}$ al momento del diagnóstico, de ahí que el embarazo sea un momento importante en la pesquisa de infectados asintomáticos, al solicitar en forma universal el test de screening en el primer control prenatal; así, en esta casuística el $35 \%$ de las pacientes descubrió su enfermedad en esta instancia.
El elemento más importante con relación al riesgo de transmisión perinatal de VIH es la carga viral materna cercana al parto $(8,9)$. En esta serie sólo el 30\% tenía carga viral indetectable o muy baja al inicio del embarazo. Pero luego de instalar tri-terapia, el 97,5\% logró ese objetivo al momento del parto. La única excepción fue una paciente con carga viral elevada, que respondió adecuadamente a la terapia, reduciendo de 187.000 a 1.900 copias $/ \mathrm{ml}$ y que era indetectable en el primer control postparto. El esquema de tri-terapia también se asocia a una mejoría en el estado inmunitario de la paciente (2), similar observación hubo en nuestra serie con la significativa elevación de los linfocitos CD4.

Las combinaciones de drogas recibidas por las pacientes son las mismas que se indican en personas no embarazadas (habitualmente 2 INTR +1 INNTR; o 2 INTR + 1 IP), con excepción de efavirenz por su efecto teratogénico en animales, y algunos que no han completado sus estudios de seguridad. El esquema más utilizado en nuestro estudio fue de 2 INTR + 1 IP $(70 \%)$, dando prioridad a zidovudine, pues fue la primera droga que demostró utilidad en la reducción de la transmisión perinatal, y con la que existe mayor experiencia $(6,11,12)$.

Considerando que: a) la transmisión perinatal se produce fundamentalmente al momento del parto o en las últimas semanas de gestación (7), b) tri-terapia es capaz de llevar la carga viral a niveles indetectables en un elevado porcentaje de pacientes a las 8-12 semanas de uso $(2,10), c)$ que los estudios de teratogenicidad, carcinogénesis y otros efectos tóxicos potenciales de la exposición in útero de dichas drogas son aún de duración limitada y d) en la primera mitad de la gestación los vómitos y náuseas dificulta la adhesión al tratamiento, favoreciendo la mono o biterapia encubierta con el desarrollo de resistencia viral, hace razonable iniciar tri-terapia en la segunda mitad del embarazo. En nuestra serie el $70 \%$ inició tri-terapia a las $24-25$ semanas, y un $97,5 \%$ del total de pacientes logró carga viral indetectable o inferior a $1000 \mathrm{copias} / \mathrm{ml}$ al momento del parto.

Existe un bajo porcentaje de intolerancia que requiera modificar los esquemas de tratamiento. Lo más frecuente fue el vómito y la diarrea $(7,5 \%)$ y en nuestra serie no hubo casos de suspensión de terapia. Tampoco se observaron otras complicaciones graves descritas como la acidosis láctica $(13,14)$.

En 1998, Lorenzi y cols publicó un estudio que 
incluía 37 pacientes embarazadas tratadas con bi o tri-terapia. El autor planteó una posible asociación entre la terapia anti-retroviral y riesgo de prematurez, pues la tasa de parto prematuro fue de $30 \%$ (15). Posteriormente, un estudio europeo que incluyó 3.920 pacientes y sus recién nacidos, entre las cuales sólo $323(8,2 \%)$ recibieron una bi o tri-terapia, mostró un aumento en el riesgo de prematurez entre los niños expuestos a una combinación anti-retroviral con o sin inhibidores de proteasa (16). Estos resultados no fueron confirmados por otros estudios. En 2000, Tuomala y cols, con una cohorte de 1.472 pacientes embarazadas, de las cuales $1.150(78 \%)$ recibieron una terapia anti-retroviral asociada, no encontraron asociación entre terapia y parto prematuro (17), mientras que la tasa de prematurez fue mayor en las pacientes infectadas que no recibieron terapia. Finalmente, un meta-análisis que consideró siete estudios clínicos, incluyendo 2.113 pacientes que recibieron algún tratamiento anti-retroviral y 1.143 pacientes infectadas no tratadas, mostró que el empleo de terapia anti-retroviral combinada no aumentaba el riesgo de parto prematuro, de bajo peso al nacer o de Apgar bajo, en comparación a las pacientes sin terapia anti-retroviral o las que recibieron monoterapia (18). Esto concuerda con una comunicación preliminar de nuestro grupo (19), y que se mantiene en el presente estudio, con una tasa de prematurez de $5 \%$.

Hubo tres casos de diabetes gestacional. Los tres en usuarias de inhibidores de proteasa, los cuales se conocen que pueden producir hiperglicemia, si bien hoy la evidencia no es concluyente entre el uso de esas drogas y el aumento de riesgo de esta patología (20).

La cesárea electiva y con membranas íntegras, permite reducir el riesgo de transmisión perinatal de $\mathrm{VIH}$ entre las que no recibieron tratamiento anti-retroviral o que recibieron monoterapia con AZT $(21,22)$. Por otra parte, el efecto protector adicional de la cesárea no está demostrado entre las pacientes que tienen una carga viral indetectable gracias al empleo de una tri-terapia anti-retroviral eficaz. En consecuencia, el American College of Obstetrics and Gynecology recomienda una cesárea electiva a las pacientes que tengan una carga viral superior a 1000 copias/ml en una muestra cercana al parto (5). En nuestra serie, si la paciente tenía carga inferior a $1000 \mathrm{copias} / \mathrm{ml}$ y sin contraindicación obstétrica se le ofrecía parto vaginal, exceptuando si el intervalo entre la rotura de membranas y el parto se estimaba mayor de 46 horas. Esta conducta logró un $50 \%$ de parto vaginal, significativamente mejor al $100 \%$ de cesárea que la población que recibe zidovudine sola.

En esta serie con la tri-terapia propuesta y ofrecer parto vaginal en las condiciones descritas, la tasa de transmisión perinatal de $\mathrm{VIH}$ fue $0 \%$, concordante con el riesgo de 0 a $2 \%$ publicado en la literatura $(9,10)$.

En conclusión, en el manejo de una embarazada $\mathrm{VIH}(+)$ conviene considerar el tratamiento antiretroviral actual y futuro de la infección materna y la profilaxis anti-retroviral para reducir el riesgo de transmisión perinatal. Actualmente, tri-terapia ofrece la menor tasa de transmisión perinatal asociado a una mayor efectividad en el control viral materno, lo que permite no comprometer futuros tratamientos. Permite además, ofrecer parto vaginal a un número significativo de pacientes, como también iniciar la terapia en la segunda mitad del embarazo, mejorando la adherencia al tratamiento, evitando la monoterapia encubierta. Sin embargo, debemos considerar que el costo del tratamiento es alto y aún no hay un seguimiento de largo plazo de los niños para algunas de las drogas utilizadas.

\section{BIBLIOGRAFÍA}

1. Gottlieb MS, Schanker MM, Fan PT. Pneumocystis pneumonia- Los Angeles. MMWR 1981; 30: 250-2.

2. CDC. Guidelines for the use of antiretroviral agents in HIV-infected adults and adolescents. MMWR 2002; 51(RR-7): 1-56.

3. Soto J. VIH / SIDA materno-infantil, es posible erradicar la infección neonatal. REV CHIL OBSTET GINECOL 2002; 67(1): 69-74.

4. Gallant JE, Eldred LJ, Leslie JM, Chaisson RE, Quinn TC. Impact of the 1993 revision of the CDC case definition on the performance of the $\mathrm{WHO}$ and $\mathrm{PAHO}$ clinical case definitions for AIDS. AIDS 1993; 7(10): 1396-7.

5. ACOG. Committe Opinion: Scheduled cesarean delivery and the prevention of vertical transmission of HIV infection. $\mathrm{N}^{\circ}$ 234, May 2000 (replaces $\mathrm{N}^{\circ}$ 219, August 1999). Int J Gynaecol Obstet 2001; 73(3): 279-81.

6. Connor EM, Sperling RS, Gelber R, Kiselev P, Scott G, O'Sullivan MJ y cols. Reduction of maternalinfant transmission of human immunodeficiency virus type 1 with zidovudine treatment. $\mathrm{N}$ Engl J Med 1994; 331(18): 1173-80.

7. Mofenson LM. Interaction between timing of perinatal human immunodeficiency virus infection and the design of preventive and therapeutic interventions. Acta Paediatr Suppl 1997; 421: 1-9.

8. Minkoff $\mathrm{H}$. Human immunodeficiency virus infection in pregnancy. Obstet Gynecol 2003; 101(4): $797-$ 810. 
9. Cooper ER, Charurat M, Mofenson L, Hanson IC, Pitt J, Diaz C y cols. Combination antiretroviral strategies for the treatment of pregnant HIV-1 infected women and prevention of perinatal HIV-1 transmission. J Acquir Immune Defic Syndr Hum Retrovirol 2002; 29(5): 484-94.

10. Morris A, Cu-Uvin S, Harwell J, Garb J, Zorrilla C, Vajaranant $\mathrm{M}$ y cols. Multicenter review of Protease Inhibitors in 89 pregnancies. JAIDS 2000; 25(4): 306-11.

11. Cooper ER, Nugent RP, Diaz C, Pitt J, Hanson C, Kalish LA, y cols. After AIDS Clinical Trial 076: the changing pattern of zidovudine use during pregnancy, and the subsequent reduction in vertical transmission of human immunodeficiency virus in a cohort of infected women and their infants. J Infect Dis 1996; 174(6): 1207-11.

12. Mayaux MJ, Teglas JP, Mandelbrot L, Berrebi A, Gallais $\mathrm{H}$, Matheron $S$ y cols. Acceptability and impact of zidovudine for prevention of mother-tochild human immunodeficiency virus-1 transmission in France. J Pediatr 1997; 131(6): 857-62.

13. Cote HC, Brumme ZL, Craib KJ, Alexander CS, Wynhoven $B$, Ting $L y$ cols. Changes in mitochondrial DNA as a marker of nucleoside toxicity in HIVinfected patients. N Engl J Med 2002; 34(11): 81120.

14. Mandelbrot L, Kermarrec N, Marcollet A, Lafanechere A, Longuet $P$, Chosidow D y cols. Case report: nucleoside analogue-induced lactic acidosis in the third trimester of pregnancy. AIDS 2003; 17(2): 2723.

15. Lorenzi P, Spicher VM, Laubereau B, Hirschel B, Kind C, Rudin C y cols. Antiretroviral therapies in pregnancy: maternal, fetal and neonatal effects. Swiss HIV Cohort Study, the Swiss Collaborative HIV and Pregnancy Study, and the Swiss Neonatal HIV Study. AIDS 1998; 12(18): F241-7.

16. THE EUROPEAN COLLABORATIVE STUDY AND THE SWISS MOTHER + CHILD HIV COHORT STUDY. Combination antiretroviral therapy and duration of pregnancy. AIDS 2000; 14(18): 2913-20.

17. Tuomala R. Interim analysis of Pediatric AIDS Clinical Trials Group Protocol 367. Presented at the Pediatric AIDS Clinical Trials Group Meeting. Crystal City, Virginia, July 2000.

18. Tuomala RE, Shapiro D, Mofenson LM, Bryson Y, Culnane M, Hughes MD y cols. Antiretroviral therapy during pregnancy and the risk of adverse outcome. N Engl J Med 2000; 346: 1863-70.

19. Abarzúa F, Hubinont $H$, Vandercam B. Highly active antiretroviral therapy in pregnant HIV-infected women. Efficacy and safety for the mothers and infants. At 9th European AIDS Conference, Warsaw October 25-29, 2003.

20. Dube MP, Sattler FR. Metabolic complications of antiretroviral therapies. AIDS Clinical Care 1998; 10(6): 41-4.

21. THE INTERNATIONAL PERINATAL HIV GROUP. The Mode of Delivery and the Risk of Vertical Transmission of Human Immunodeficiency Virus Type 1- a Meta-Analysis of 15 Prospective Cohort Studies. N Engl J Med 1999; 340(13): 977-87.

22. THE EUROPEAN MODE OF DELIVERY COLLABORATION. Elective cesarean-section versus vaginal delivery in prevention of vertical HIV-1 transmission: a randomised clinical trial. Lancet 1999; 353(9158): 1035-9. 